\title{
Análisis de la vulnerabilidad por los deslizamientos en masa, caso: Tlacuitlapa, Guerrero
}

\author{
Rosalva Pérez-Gutiérrez \\ Unidad Académica de Ciencias de la Tierra, Universidad Autónoma de Guerrero, \\ Ex - Hacienda de San Juan Bautista, Taxco El Viejo Guerrero, 40200. \\ perezr@geologia.unam.mx
}

\section{Resumen}

En el poblado de Tlacuitlapa, norte del Estado de Guerrero, se tienen problemas de inestabilidad geológica como producto de deslizamientos en masa y la consiguiente afectación en mayor o menor grado del patrimonio de varias familias. A fin de determinar la vulnerabilidad de la zona se llevó a cabo la cartografía geológica que incluyó la ubicación y caracterización de áreas afectadas por deslizamiento de bloques de roca y suelo. De manera paralela a la caracterización geológica del sitio, se elaboró un censo el cuál aportó información valiosa acerca de los daños producidos en la infraestructura de la comunidad.

Como resultado del análisis se definieron zonas con grados de vulnerabilidad distintos. Para la familias que se encuentran dentro de la zona de riesgo no mitigable, 32 familias hasta el momento, se recomienda el desalojo de sus propiedades a fin de evitar pérdidas humanas. En el caso de la zona de riesgo parcialmente mitigable, es importante realizar una valoración detallada de los daños e implementar obras de ingeniería que permitan minimizar del riesgo.

Los deslizamientos de material son el producto de la interacción entre la pendiente del terreno, el suelo y el depósito de talud sobre el que se asentó la población. Se considera que el principal factor detonante es el agua de lluvia, aunque no se descarta que la sismicidad de la región del Río Balsas pueda tener alguna repercusión.

Palabras clave: deslizamientos en masa, vulnerabilidad, afectaciones, Tlacuitlapa.

\begin{abstract}
In the town of Tlacuitlapa, in the northern part of the state of Guerrero, geological instability resulting in landslides has affected, to a greater or lesser degree, homes and livelihoods. Geological mapping was used to determine the vulnerability of the zone and included the location and nature of the areas affected by landslides involving rock blocks and earth. In parallel with the geological characterization a survey was conducted, which provided valuable information about the damage caused to the community's infrastructure.

The analysis defined zones with different degrees of vulnerability. People living within the zone where risks cannot be mitigated-32 families so far-were advised to abandon their properties in order to avoid possible human losses. In the case of the zone where danger might be partially mitigated, a detailed evaluation of potential damage is required, with engineering works to minimize the risk.

The landslides are the result of the interaction of the slope of the location, the soil and the deposits on which the town was founded. Rainfall is considered to be the main trigger for the landslides, although the impact of the seismic nature of the Balsas River basin cannot be ruled out.
\end{abstract}

Key words: landslides, vulnerability, impact, Tlacuitlapa. 


\section{Introducción}

En la búsqueda de satisfacer necesidades primarias, como el abasto de agua, muchas veces las personas establecen sus comunidades en sitios vulnerables (zonas escarpadas, planicies de inundación, etc). El poblado de Tlacuitlapa, se encuentra asentado en la parte alta de la ladera oriental de la Sierra de Chilacachapa, norte del Estado de Guerrero (Figura 1). La ladera tiene una pendiente abrupta de $39^{\circ}$ y en ella en los últimos 10 años se han tenido deslizamientos en masa que han generado daños materiales en distinto grado en las propiedades de varias familias. Los deslizamientos con mayor repercusión en la población se produjeron en los meses de septiembre y octubre del 2004, después de una temporada de lluvias con una cantidad de precipitación alta. Desafortunadamente, no se cuenta con el valor cuantitativo de la precipitación para ese periodo de tiempo, debido a que la estación metereológica más cercana se ubica en la ciudad de Iguala, donde las características climáticas son muy distintas. En esa ocasión, se produjo el deslizamiento pendiente abajo de bloques con dimensiones considerables de hasta $36 \mathrm{~m} 3$, que destruyeron dos inmuebles y generaron el agrietamiento y basculamiento de varias viviendas, así como de la calle principal. Adicionalmente, para el mismo periodo en la zona del Río Balsas se tuvo la ocurrencia de varios sismos, destacando el del 28 de octubre a las 15:30 pm con una magnitud de 4.6 grados, profundidad de $51 \mathrm{~km}$ y un epicentro ubicado en las coordenadas geográficas: latitud $18.34^{\circ}$ y longitud $-97.74^{\circ}$ (Servicio Sismológico Nacional, 2004), es decir, a unos cuantos kilómetros al norte de Tlacuitlapa. Este sismo produjo una mayor abertura y extensión de las fracturas ya existentes, así como la aparición de nuevo fracturamiento. Es evidente que por las características del sitio mencionadas arriba, el deslizamiento en masa va a continuar y por consiguiente, generará afectaciones mayores. Lo anterior, motivó la realización de este trabajo geológico cuya aportación principal es el mapa de vulnerabilidad para el poblado de Tlacuitlapa.

\section{Metodología}

El análisis inició con el reconocimiento de áreas afectadas por deslizamiento dentro del poblado y en sus alrededores. Posteriormente, se cartografiaron y determinaron las características de los diferentes paquetes de rocas y los deslizamientos. Como base para la cartografía se utilizó la carta topográfica Teloloapan E14A77 escala 1:50 000

a)
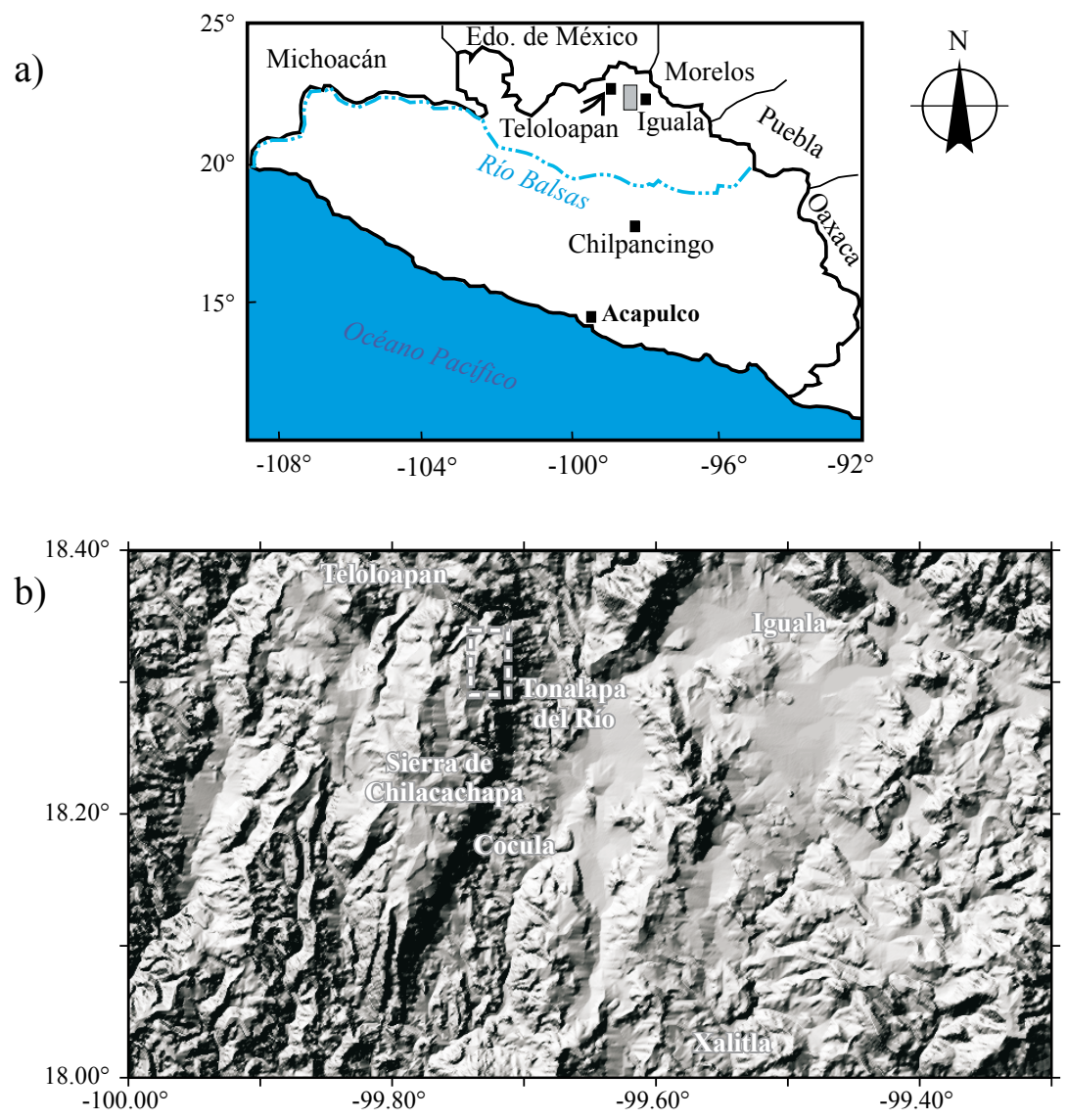

Figura 1. Localización de la zona de estudio a) en el estado de Guerrero y b) en un modelo de elevación de la región, en el que se aprecian distintos rasgos morfológicos. 
(INEGI, 1998). De manera paralela a la caracterización geológica del sitio se elaboró un censo, el cuál aportó información valiosa acerca de la cantidad y características de los daños producidos a la infraestructura.

Como resultado del trabajo geológico se obtuvieron dos mapas: 1) el mapa geológico, en el cuál se plasmó la distribución de los diferentes paquetes de rocas y sus relaciones, y 2) el mapa de vulnerabilidad, que incluye la ubicación de fracturas y deslizamientos que generaron afectaciones, y en el que se definen zonas con diferentes grado de vulnerabilidad que fue determinado básicamente por los daños que han sufrido viviendas y calles.

\section{Aspectos geomorfológicos}

El poblado de Tlacuitlapa se ubica en la ladera oriental de la Sierra de Chilacachapa que tiene una orientación general N-S. La parte superior está formada por una meseta que está limitada al oriente por un escarpe que en su base tiene una estrecha zona de depósito topográficamente semiplana denominada La Joya (Figura 2) a partir de ésta, hacia el oriente inicia una ladera que desciende desde 1300 hasta $700 \mathrm{msnm}$ con pendientes que alcanzan los $39^{\circ}$.

En la parte topográficamente alta del poblado no se tiene el desarrollo de una red de drenaje superficial, lo cuál refleja alta permeabilidad de la zona, ya que en temporada de lluvias el curso del agua de los manantiales y el agua de la lluvia es principalmente a través de depósitos de bloques no consolidados.

El pueblo está asentado en la base y sobre del depósito de talud, ya que una cantidad importante de esta agua resurge en dos manantiales ubicados dentro del depósito (Figura 3), siendo el más importante el que se encuentra en las cercanías de la Escuela Primaria Cristóbal Colón. Hay cuatro manantiales más en las inmediaciones del poblado, pero estos no están asociados con el depósito de talud. El

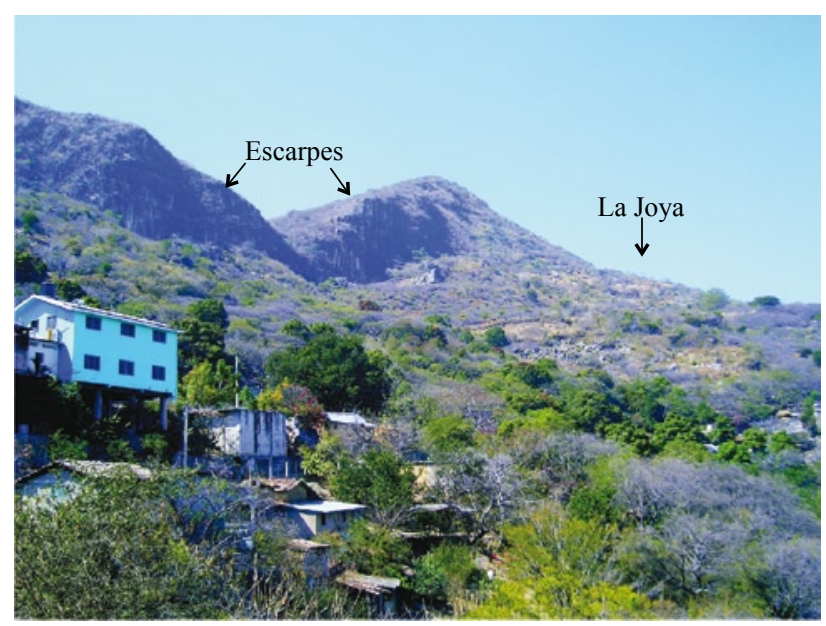

Figura 2. Imagen que muestra las características morfológicas de la parte alta de Tlacuitlapa, Guerrero. agua se utiliza principalmente para consumo humano y tareas domésticas.

\section{Geología}

Las rocas que afloran en la zona de estudio pertenecen principalmente a las formaciones Morelos y Mexcala (Fries, 1960), depósito de talud y roca intrusiva (Figura 3). Las rocas de la Formación Morelos afloran en la porción occidental de la zona de estudio cubriendo un $25 \%$ del total del área (Figura 3), su expresión topográfica es casi plana y está limitada al oriente por un escarpe de 50 a 80 $\mathrm{m}$ de altura. Está formación está compuesta por calizas arrecifales y dolomías de color gris claro que presenta estratificación gruesa a masiva de 0.20 hasta $3 \mathrm{~m}$ de espesor y laminaciones. La textura de las rocas varía de mudstone a wackstone, en algunas partes presenta rasgos de disolución y nódulos de pedernal de color negro. A partir de su contenido fósil a esta unidad se le ha determinado una edad Albiano-Cenomaniano (Fries, 1960; Morales, 1989; Guerrero et al., 1991).

Los afloramientos de la Formación Mexcala se localizan en la porción centro y oriental, cubriendo un $60 \%$ del total del área. Topográficamente conforma una serie de laderas principalmente orientadas este-oeste con inclinación mayor de $30^{\circ}$. Consiste en una sucesión de capas interestratificadas de lutitas, areniscas y areniscas calcáreas de grano fino a medio. El color de estas rocas es generalmente ocre y se encuentran formando capas de espesor variable entre 5 y $40 \mathrm{~cm}$ (Figura 4). La edad de éstas rocas se considera Turoniano-Campaniano (Fries, 1960; Ontiveros, 1973). De manera general, está formación se encuentra intensamente plegada. En la región la relación estratigráfica respecto a las rocas de la Formación Morelos, es de sobreposición por discordancia litológica; sin embargo, en la zona de estudio la relación es tectónica, es por medio de una cabalgadura que produce que las rocas de la Formación Morelos estén sobre las rocas de la Formación Mexcala (Figura 5).

En la parte noreste del área aflora una roca intrusiva (Figura 3) que topográficamente constituye una loma alargada y plana. Es una sienita de color gris verde de estructura compacta pero superficialmente muy intemperizada. Presenta una textura fanerítica de grano muy fino constituida por cristales de plagioclasa, hornblenda y augita incluidos en una matriz cristalina. Esta roca corta a la caliza de la Formación Morelos y a la lutita-arenisca de la Formación Mexcala.

La unidad más joven corresponde a un depósito de talud que aflora en la porción central del área de estudio (Figura 3), constituido por bloques que han resultado del intemperismo y erosión de las rocas de la Formación Morelos. El depósito no está consolidado, presenta una mezcla de bloques de caliza y dolomías de color gris claro a gris oscuro, con forma subangulosa a subredondeada y tamaño variable de 1 a $10 \mathrm{~m}$ de diámetro (Figura 6), que 


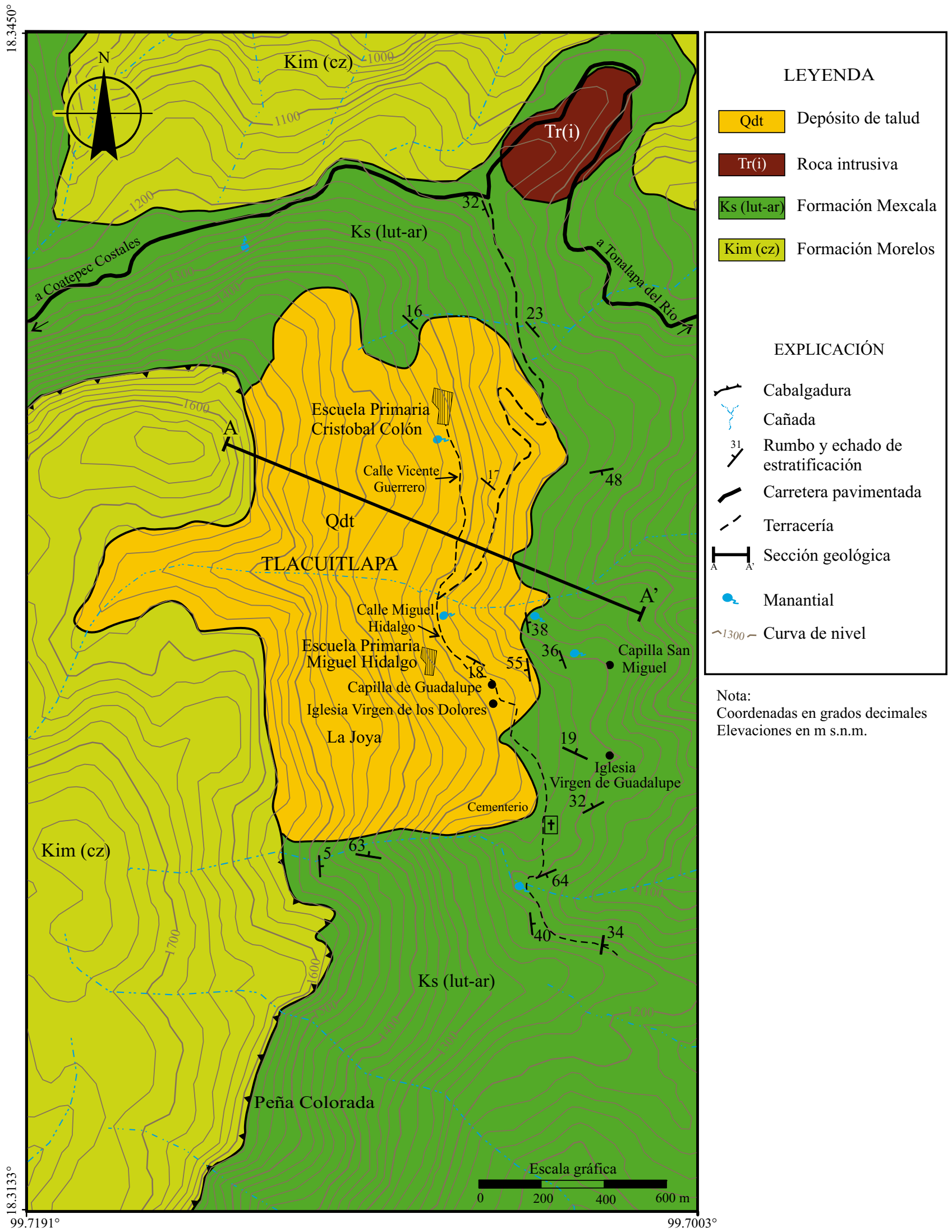

Figura 3. Mapa geológico que muestra la distribución de los paquetes litológicos en el área de Tlacuitlapa, Guerrero. 


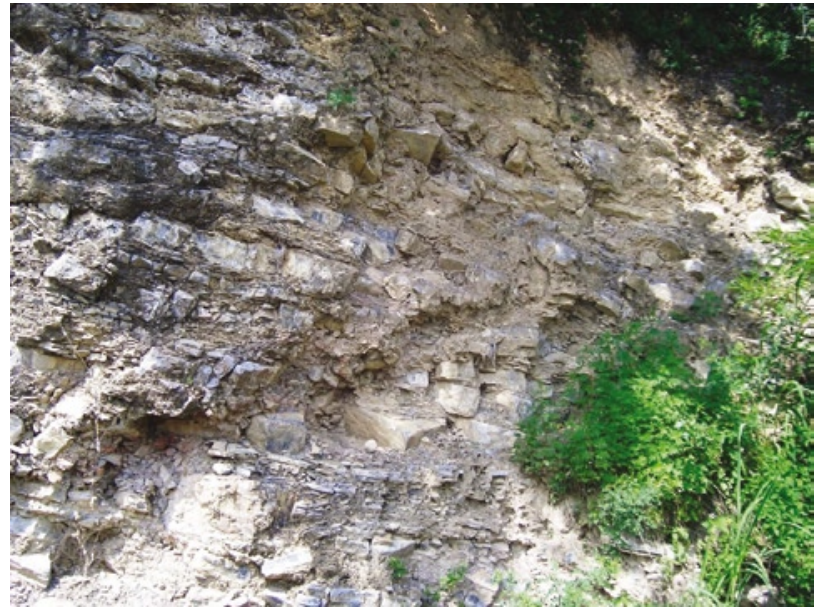

Figura 4. Capas de lutitas y areniscas calcáreas de la Formación Mexcala en el camino de acceso a Tlacuitlapa, las dimensiones del afloramiento son de $10 \times 6 \mathrm{~m}$.

están inmersos en una matriz compuesta por suelo deleznable y fácilmente erosionable por el agua superficial. Este depósito se encuentra sobre las rocas de la Formación Mexcala. Dentro de esta unidad se tiene la presencia de los principales manantiales y como consecuencia de esto, una parte importante del poblado de Tlacuitlapa se estableció sobre ella (Figura 5).

La distribución del suelo es irregular en el área de estudio por lo que no se representa en el Mapa Geológico. Consiste de material residual producto de la intemperización principalmente de las rocas de la Formación Mexcala, es de color ocre y su espesor varía de unos cuantos centímetros hasta $5 \mathrm{~m}$ (Figura 7). Se sobrepone a las rocas de la Formación Mexcala y en el caso del depósito de talud, el suelo funciona como matriz, aunque éste es deleznable.

\section{Estructuras}

La estructura geológica más importante de la zona, la constituye la cabalgadura de la Formación Morelos sobre la Formación Mexcala, que genera una posición estratigráfica invertida entre estas dos formaciones. Esta estructura tiene una orientación norte-sur y una inclinación hacia el noroeste (Figura 5), forma parte del Cinturón de Pliegues y Cabalgaduras Mexicano dentro del Sector Teloloapan (Campa y Coney, 1983; Campa, 1985; Pérez-Gutiérrez, 1993). La expresión morfológica de esta estructura es un escarpe de 50 a $80 \mathrm{~m}$ de altura, que es la fuente de los bloques del depósito de talud. La superficie de estratificación de las calizas de la Formación Morelos presenta un

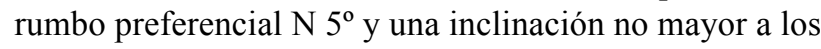
$20^{\circ}$ hacia el sureste.

La estratificación de las rocas de la Formación Mexcala es media a delgada con estratos de 5 a $30 \mathrm{~cm}$ de espesor y una orientación de las capas que varía de $302^{\circ} / 20^{\circ}, 359^{\circ} / 25^{\circ}$ y $159^{\circ} / 34^{\circ}$ debido al desarrollo de un plegamiento complejo. Los pliegues presentan variaciones geométricas de inclinados a recumbentes; su vergencia es hacia el oriente y los ejes tienen una orientación preferencial N-S.

Se determinaron tres sistemas de fracturamiento que cortan a las rocas de la Formación Mexcala, sus orientaciones son $281^{\circ} / 73^{\circ}, 339^{\circ} / 58^{\circ}$ y $20^{\circ} / 87^{\circ}$. La intersección o relación de distintos sistemas de fractura delimitan bloques de roca con geometría y tamaño variable.

\section{Afectaciones}

Las afectaciones que se tienen hasta ahora en el poblado de Tlacuitlapa consisten principalmente de fracturamiento y fallamiento del terreno, producto del deslizamiento de

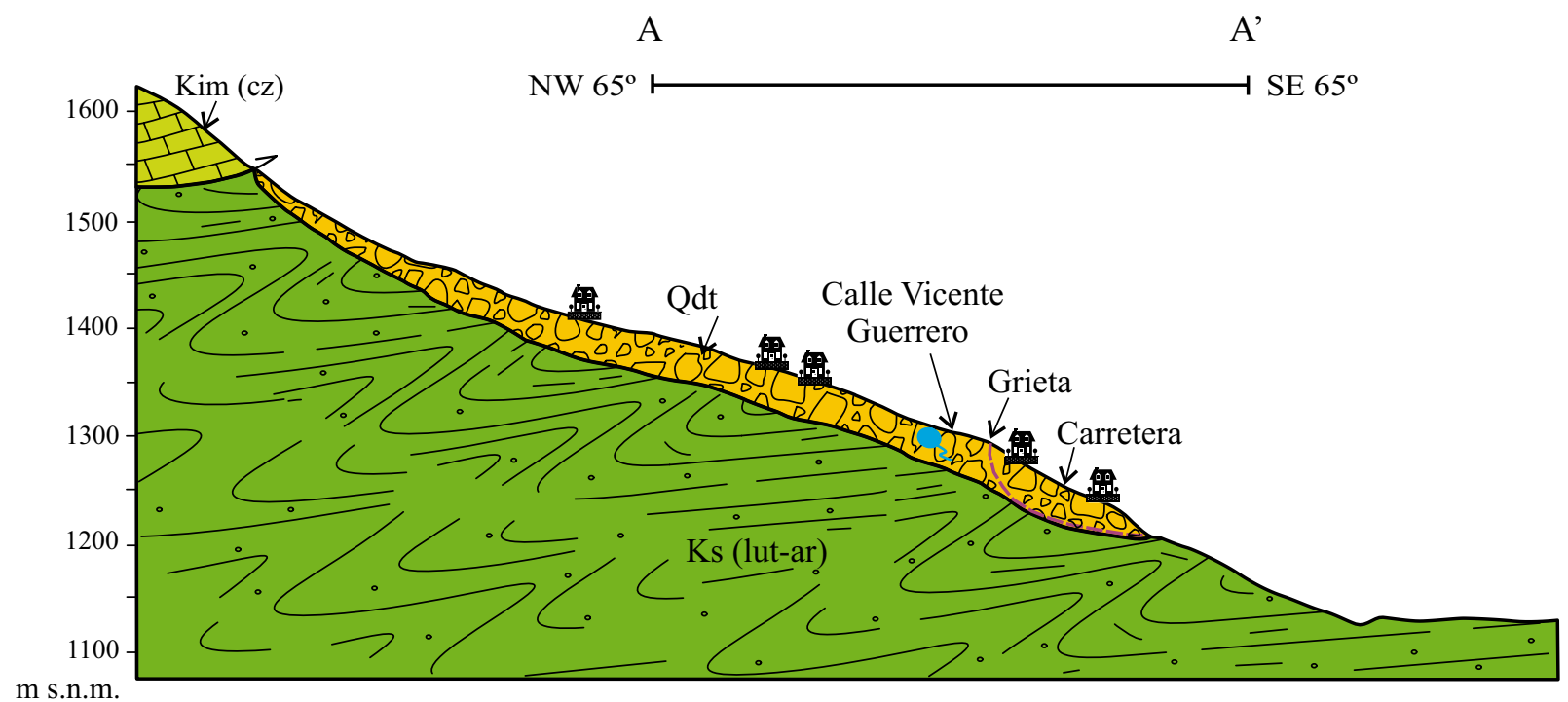

Figura 5. Sección geológica que muestra la relación entre los distintos paquetes litológicos y la disposición del poblado. Kim (cz): Formación Morelos; Ks (lut-ar): Formación Mexcala; Qdt: Depósito de talud. 


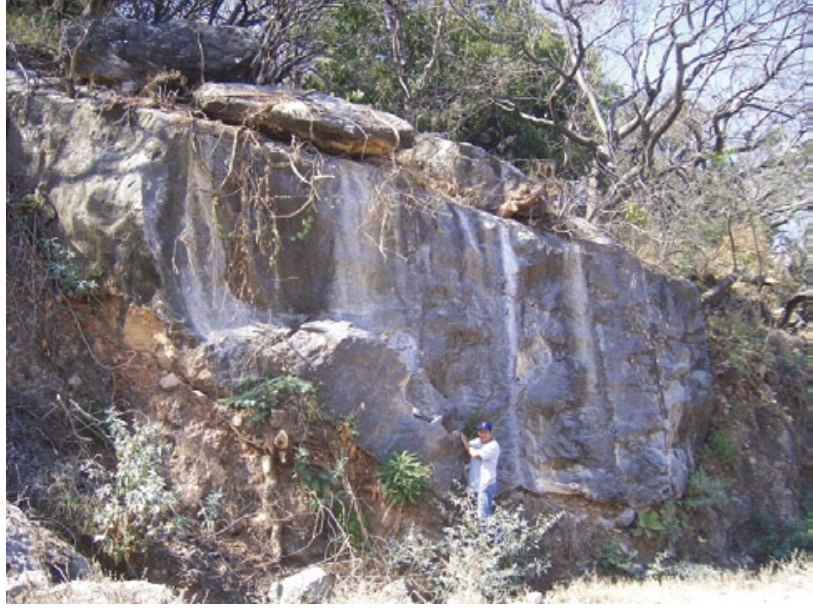

Figura 6. Bloque de caliza que forma parte del depósito de talud en el camino de acceso a Tlacuitlapa, sus dimensiones son de $5 \times 8 \mathrm{~m}$ aproximadamente.

los materiales no consolidados a lo largo de su contacto con las rocas consolidadas.

Se identificaron nueve deslizamientos, distribuidos en cuatro de las cañadas que disectan la ladera y con una dirección de movimiento preferencialmente hacia el oriente (Figura 12), en su mayoría tienen formas alargadas y sus dimensiones varían entre 1352 y $48205 \mathrm{~m}^{2}$ con espesor promedio de $1 \mathrm{~m}$. Uno de los delizamientos, el de dimensiones mayores, está asociado al depósito de talud, en el resto de los deslizamientos el material involucrado es suelo y la lutita-arenisca de la Formación Mexcala.

De manera general, se trata de movimientos rotacionales donde las fracturas y fallas relacionadas a los deslizamientos de bloques presentan una geometría semicircular con una orientación preferencial noreste-suroeste y una longitud aproximada de $100 \mathrm{~m}$ y en algunos casos un desplazamiento vertical que alcanza los $1.50 \mathrm{~m}$. La abertura de las fracturas ha variado de manera continua; por ejemplo, una fractura

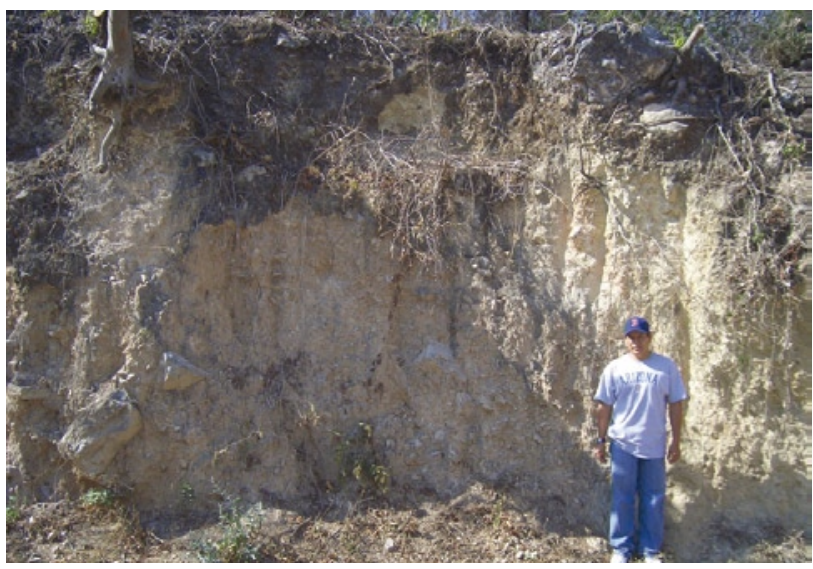

Figura 7. Afloramiento que muestra un espesor de $5 \mathrm{~m}$ de suelo.

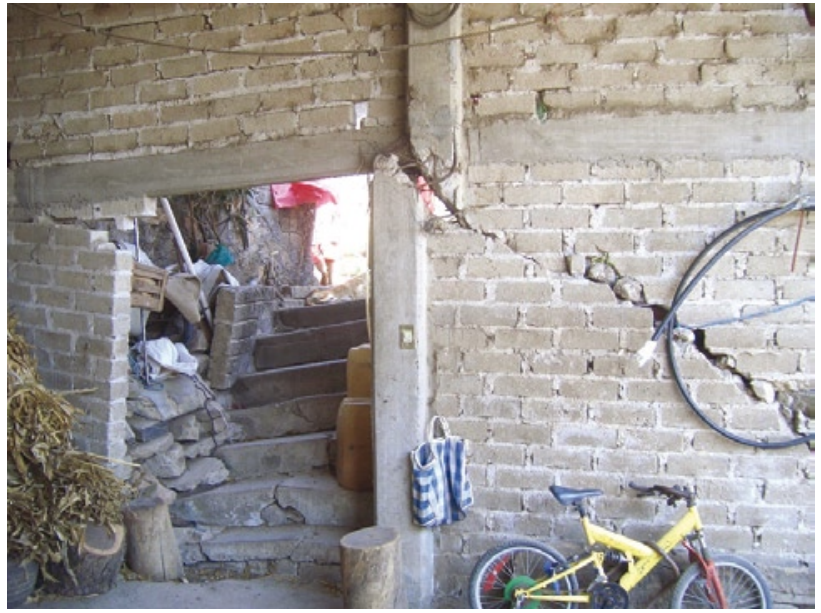

Figura 8. Imagen que muestra los daños producidos en una pared, fracturamiento, el castillo ha sido roto y la varilla de acero de 1" doblada, ancho de la puerta $1.5 \mathrm{~m}$.

con abertura inicial de $10 \mathrm{~cm}$, dos semanas después alcanzó $36 \mathrm{~cm}$ de abertura, que equivale a un desplazamiento de 1.8 $\mathrm{cm}$ por día. La información proporcionada por los afectados indica que el surgimiento y crecimiento de las fracturas ha sido relativamente rápido y ha coincidido con el final de la temporada de lluvias.

El paso de la traza de las fracturas por casas habitación ha dado lugar al fracturamiento de cimientos, pisos y paredes (Figura 8), y en cuatro casos en particular al deslizamiento de las mismas, generando pérdidas materiales importantes.

La construcción de algunas habitaciones de las viviendas sobre bloques de roca hace a estos inmuebles más susceptibles a afectaciones. En el caso de la imagen de la Figura 9, una habitación y una escalera se edificaron sobre un bloque de aproximadamente $5 \mathrm{~m}$ de diámetro, el cuál rodó cuando el desplazamiento de una fractura ubicada en su base produjo inestabilidad en el suelo, teniendo como consecuencia la destrucción parcial de la vivienda.

Otros deslizamientos se localizan en la zona central y sur del área, principalmente en la parte topográficamente inferior del poblado. Son movimientos rotacionales que generan fracturamiento circular el cuál se ha ido propagando y el material involucrado en este caso es suelo poco consolidado, producto de la erosión de las lutitas y areniscas de la Formación Mexcala. Su ubicación y geometría se relaciona a las cañadas naturales del terreno y llegan a tener un longitud de 50 a $300 \mathrm{~m}$ (Figura 10).

\section{Población afectada}

De manera simultánea al levantamiento de los rasgos geológicos de la zona de estudio se realizó un censo de la población afectada. Este censo permitió obtener un panorama general de las características como son número 


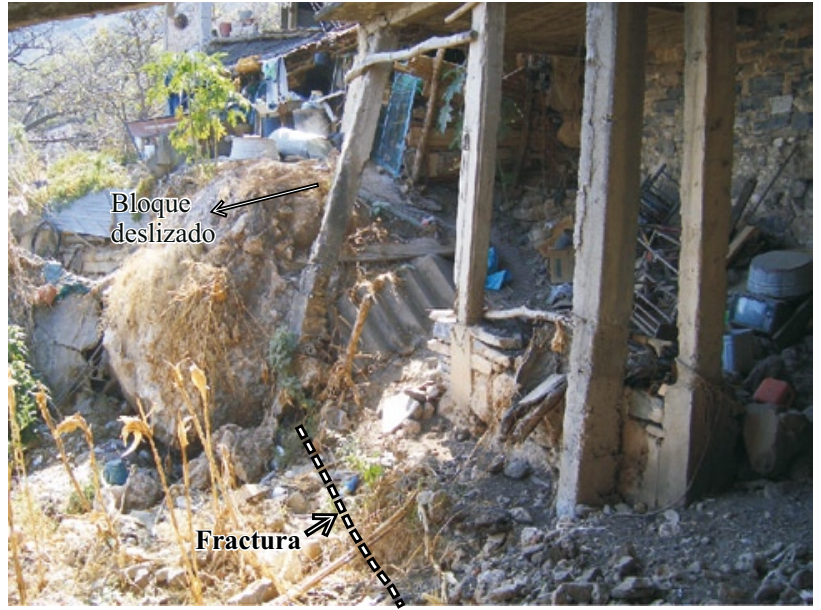

Figura 9. Fotografía que muestra como el desplazamiento de un bloque de grandes dimensiones generó daños severos en una vivienda.

de familias afectadas, materiales de construcción de los inmuebles y los daños sufridos.

La población total de Tlacuitlapa es de 917 personas (INEGI 2005), a la fecha se encuentran afectadas 121 personas, distribuidas en 32 familias. Las características de los materiales con que están construidos los inmuebles afectados son predominantemente paredes de tabique y en menor proporción adobe, techos predominantemente de loza y pisos de cemento.

En las gráficas de la Figura 11 se aprecian el tipo de afectación que han sufrido los inmuebles; hasta ahora los daños mayores son agrietamientos; sin embargo, el grado de las afectaciones puede aumentar sí los deslizamientos de material continúan.

La calle Vicente Guerrero, que comunica a la parte norte de Tlacuitlapa, ha sido la más afectada. El paso de la fractura más continua ha ocasionado un desplazamiento vertical de ésta de hasta $70 \mathrm{~cm}$. La distribución de agua y el servicio de drenaje no se han visto afectados, debido a que los materiales que se utilizan para tales fines son mangueras de plástico y tubería de PVC, respectivamente.

\section{Resultados}

La estimación de la distribución de riesgo o vulnerabilidad generada por la interacción entre los deslizamientos y los elementos afectado tiene como finalidad proporcionar un mapa que describa de manera cualitativa o cuantitativa el nivel o grado de vulnerabilidad para una localidad (Dai et al., 2002). Considerando lo anterior, para el área de Tlacuitlapa se determinaron tres zonas con distinto grado de vulnerabilidad (Figura 12) y que son descritas a continuación:

Zona de riesgo no mitigable: esta zona se localiza en la porción central del poblado de Tlacuitlapa, es el área con afectaciones mayores que incluye a viviendas, las calles Vicente Guerrero, Miguel Hidalgo y la carretera de acceso al pueblo. La población edificó 61 viviendas sobre bloques del depósito de talud, actualmente 22 casas están involucradas en la dinámica del deslizamiento mayor y se están deslizando hacia las zonas topográficamente más bajas. Además, en ella se encuentran las fracturas con mayor continuidad $(\sim 300 \mathrm{~m})$, con aberturas de 6 a $36 \mathrm{~cm}$ y deslizamientos verticales de hasta $70 \mathrm{~cm}$.

Las viviendas involucradas están construidas principalmente con paredes de tabique, techos de losa y pisos de cemento, lo cuál implica un peso importante de estos inmuebles sobre el material en el que fueron edificados. Los cimientos de la totalidad de las viviendas no se aprecian, sin embargo, se observaron casos donde éstos no son lo suficientemente profundos como para alcanzar roca firme y de esta manera cumplir su función de fijar la construcción a la roca (Figura 13), o que se colocaron entre bloques del talud que ya sufrieron deslizamiento y por consiguiente, provocaron daños importantes en la construcción (Figura 9).

Otro factor de riesgo para esta zona, es la amenaza del deslizamiento de bloques de dimensiones variables que se encuentran descansando en una porción topográficamente más elevada. Como se mencionó en los aspectos morfológicos, en la base del escarpe se encuentra una zona semiplana en la que se tiene el depósito de los bloques generados por la erosión del escarpe, a la que los pobladores de Tlacuitlapa denominan La Joya. Al pie de La Joya se ubica una buena parte del poblado (Figura 14) y entre los inmuebles más importantes se encuentran el jardín de niños, las escuelas primarias y la secundaria. Esta porción de Tlacuitlapa se considera como parte de la zona de riesgo por la alta probabilidad del movimiento cuesta abajo de los bloques por la acción de factores detonantes como la sismicidad y el agua de lluvia.

Las afectaciones observadas en ésta zona considerada

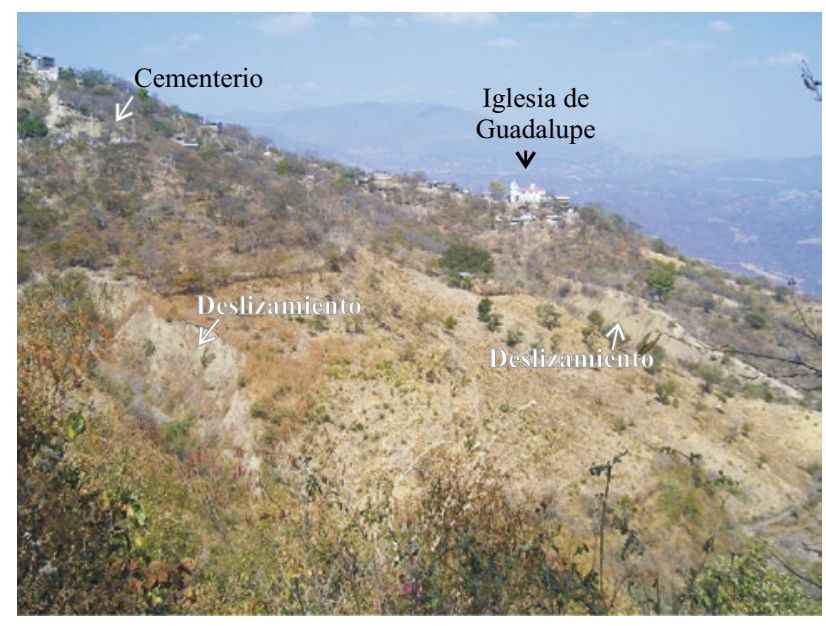

Figura 10. Deslizamientos de suelo que se ubican en la porción sur de Tlacuitlapa. 
Fracturamiento

a)

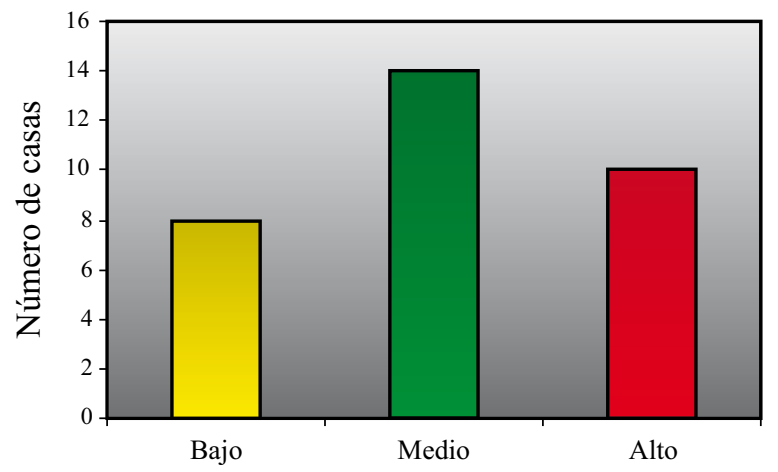

Fallamiento

b)

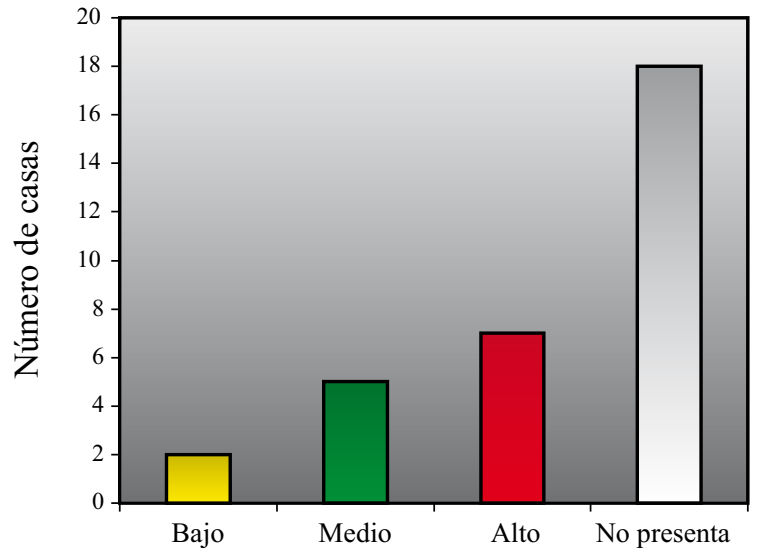

Figura 11. Gráficas que muestran los tipos y grado de afectación que presentan los inmuebles censados; a) agrietamiento, y b) deslizamiento vertical.

de alto riesgo son la respuesta de un proceso continuo de deslizamiento de material no consolidado hacia zonas topográficamente bajas. Dentro de esta zona se incluyen casas que hasta ahora no muestran afectaciones, sin embargo, se encuentran dentro del área de influencia del deslizamiento, por lo que, tarde o temprano estas viviendas se integrarán a la dinámica del mismo.

La gravedad de los daños producidos en los inmuebles y la alta probabilidad de que se acrecenten cada día, plantea la necesidad del desalojo y reubicación de la población afectada, que por ahora suma 32 familias.

Zona de riesgo parcialmente mitigable: esta zona incluye al resto de la población que está sujeta a diferente grado de vulnerabilidad, ya sea por las características del material sobre el cuál cimentaron sus viviendas o por el crecimiento de los deslizamientos de suelo.

En la porción sur de esta área se encuentran deslizamientos de material, principalmente suelo, de dimensiones variables y fracturamiento con geometría semicircular asociado a ellos. Los deslizamientos más importantes se ubican en la porción sur de la zona de estudio, en las inmediaciones del Cementerio y la Iglesia de Guadalupe (Figura 12). Hasta ahora estos deslizamientos rotacionales no han provocado daños a la población, sin embargo, es claro que de continuar el crecimiento y nueva generación de fracturas asociadas a estos deslizamientos, éstas podrían afectar al Cementerio y a las familias que habitan en el extremo sur de Tlacuitlapa.

La porción norte del poblado está asentado sobre depósito de material no consolidado y hasta ahora no han tenido problemas de deslizamiento, pero no se descarta puedan ocurrir a futuro, principalmente por la alta pendiente del terreno y la cantidad de agua que puede circular a través de los bloques.

Esta zona se considera de riesgo parcialmente mitigable, ya que pueden existir casos en los que los inmuebles no sean susceptibles a afectaciones o puedan implementarse medidas que permitan prevenir o remediar daños. La discriminación de estos sitios y las medidas de prevenciónremediación que puedan adoptarse deben ser determinadas con un estudio más detallado y puntual.

Zona de bajo riesgo: en el área de estudio se identificaron dos zonas que por su baja pendiente, entre $10 \mathrm{y} 15^{\circ}, \mathrm{y}$ el no estar directamente asociadas al depósito de talud se consideran seguras para la reubicación de la población más afectada. La primera, está en la porción occidental del área de estudio, en la parte topográficamente más elevada que morfológicamente representa una meseta. Está formada por rocas de la Formación Morelos que son muy permeables por lo que el agua de lluvia se filtra y no genera corrientes de agua superficial. En caso de reubicación de la población a esta zona, sería necesario considerar el suministro de agua mediante la perforación de pozos o el bombeo de la misma a partir de los manantiales de Tlacuitlapa. La otra zona segura se ubica al noreste, cerca del entronque de los caminos de acceso a Tlacuitlapa y a Coatepec Costales, topográficamente está formada por dos lomas alargadas con cimas casi planas, una de ellas constituida por calizas y la otra por roca intrusiva. La principal limitante de estas áreas es su tamaño, ya que sólo unas cuantas familias podrían ser reubicadas en este sitio.

\section{Interpretación}

Tomando en cuenta la información expuesta, se considera que en los deslizamientos de material en Tlacuitlapa existen dos mecanismos principales: 1) material de cada libre o desprendimiento y su depósito al pie de una ladera escarpada, en donde la gravedad es la fuerza detonante (Coch, 1995; Nelson, 2000). 2) deslizamientos rotacionales, generados a lo largo del contacto entre las rocas de la Formación Mexcala, y las rocas no consolidadas (depósito de talud y suelo), donde el material meteorizado o suelto no puede mantener la misma pendiente de la roca (Dai et al., 2002).

En el caso del material de caída libre, la presencia de discontinuidades dentro de las rocas de la Formación Morelos aunado a la pendiente escarpada, permiten a la 


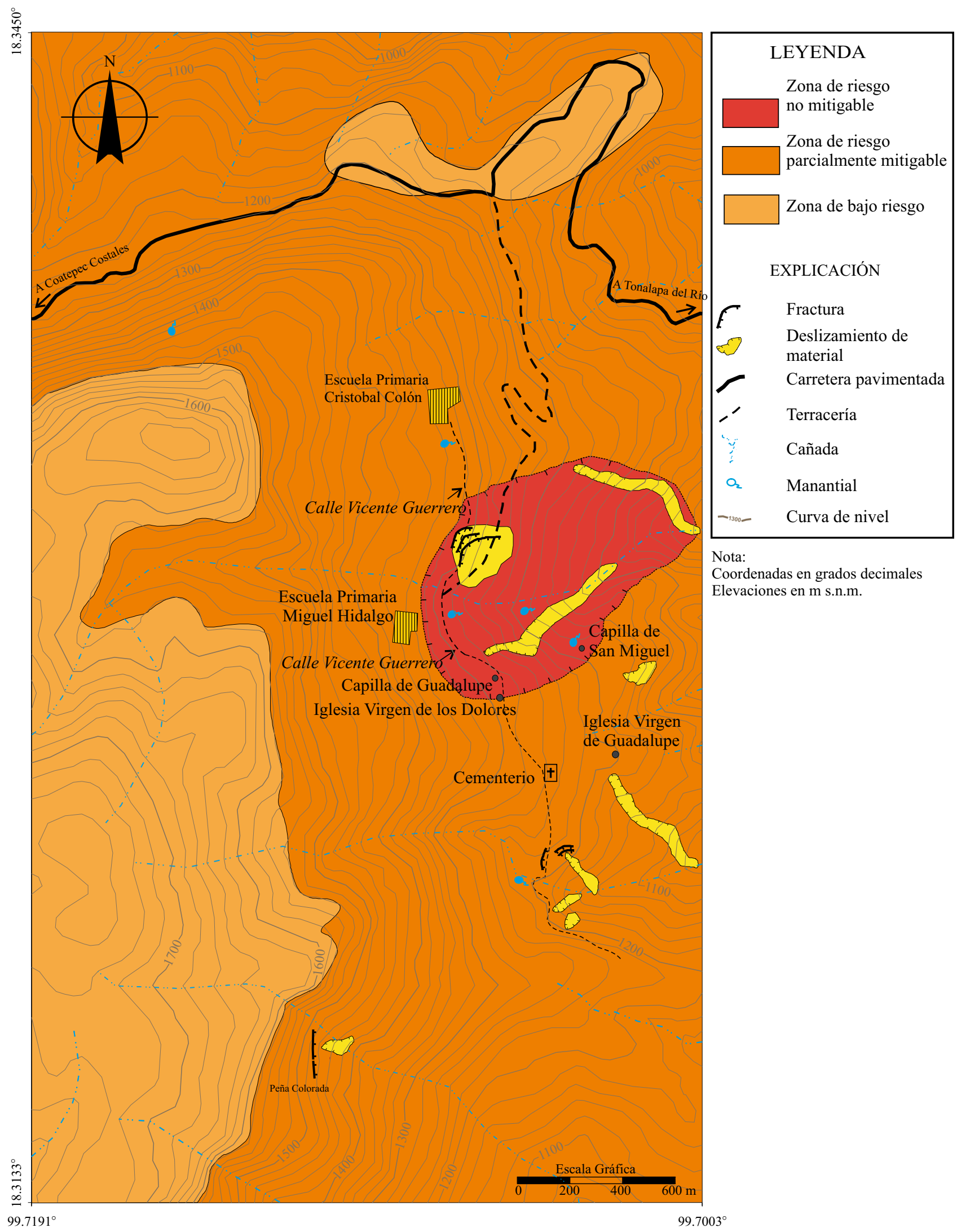

Figura 12. Mapa de vulnerabilidad para el poblado de Tlacuitlapa, Guerrero. 


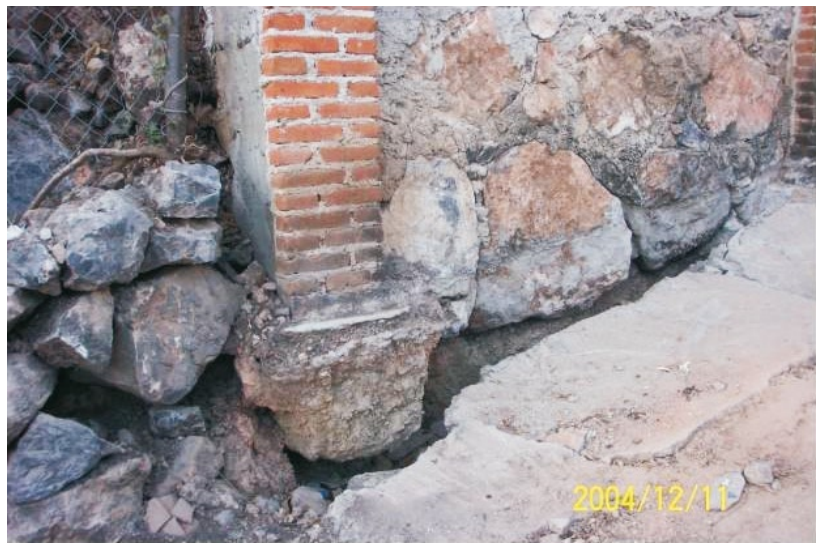

Figura 13. Imagen que muestra como el cimiento de la vivienda no está desplantado dentro de la roca sobre la que se construyó.

roca romperse en bloques de dimensiones variables que se mueven con mayor facilidad hacia abajo (Coch, 1995; Nelson, 2000).

En los deslizamientos rotacionales, además de que el principal movimiento es en la superficie de contacto entre paquetes con distinto grado de cohesividad y permeabilidad, se tiene dentro de los materiales no consolidados la presencia de espacios entre las partículas y bloques que permiten el paso de agua a través de ellos, disolviendo o arrastrando el material cementante, reduciendo la cohesión entre las partículas (Coch, 1995). En deslizamientos donde se involucran materiales no consolidados, como es el caso de los deslizamientos de Tlacuitlapa, no existe un patrón definido por la estructura de la roca y la superficie de falla es libre para encontrar la línea de menor resistencia a través de la ladera y como resultado las fallas presentan geometría circular (Hoek y Bray, 1996).

Es importante subrayar que la estructura de las rocas de la Formación Mexcala no tiene influencia directa en los deslizamientos, a pesar de que la intersección de las diferentes discontinuidades genera cinco familias de bloques con geometría y tamaño variables, y que dos de estas familias tienen dirección de salida hacia la ladera, ya que la superficie de salida de éstos no rebasa el ángulo necesario para romper el equilibrio y deslizarse.

Los principales factores que influyen en los deslizamientos de masas analizados, son las diferencias de cohesión y permeabilidad entre el material no consolidado y las rocas de la Formación Mexcala, la inclinación del terreno y la cantidad de agua de lluvia que circula a través de los bloques del depósito de talud, generando el arrastre del material que une a los bloques, y el consecuente rodamiento por gravedad de los mismos bloques ladera abajo. Por otro lado, no debe descartarse la sismicidad regional que puede producir vibración en el terreno y disparar el movimiento de material.

Las casas, al ser estructuras rígidas, registran los estadios tempranos del movimiento del material en su base

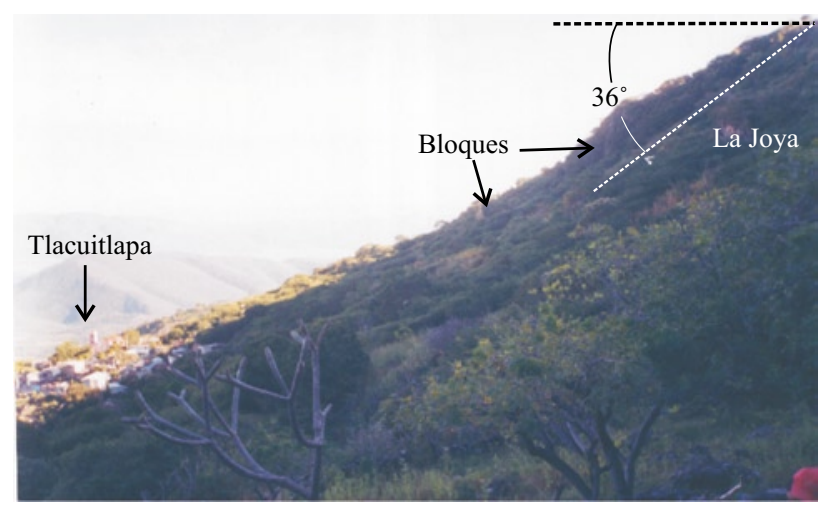

Figura 14. Paisaje en el que se aprecia los bloques que se encuentran en el paraje denominado La Joya que se ubica topográficamente arriba de Tlacuitlapa, en este punto la pendiente del terreno es de $36^{\circ}$.

(Burns et al., 2001), un movimiento continuo que puede ser muy lento u ocurrir súbitamente a veces con resultados catastróficos (Nelson, 2000); por lo que es importante monitorear el incremento en la extensión o gravedad de los daños. Una vez iniciado del proceso de deslizamiento, la fuerza de gravedad también contribuye al deslizamiento del material, sin importar la presencia de agua, y el peso de las construcciones se suma a la fuerza de atracción cuesta abajo.

En la zona de riesgo mitigable, los daños son importantes y se consideran no reparables. La implementación de obras de ingeniería encaminadas a frenar estos daños implicaría un alto costo y éstas en lugar de remediarlos, podrían inducir un mayor y rápido deslizamiento del material que causaría afectaciones más severas.

\section{Conclusiones y sugerencias}

Los paquetes litológicos que se encuentran en la zona de estudio corresponden a calizas, lutitas-areniscas, materiales no consolidados (depósito de talud y suelo) y roca ígnea intrusiva. Una buena parte del poblado se encuentra asentado sobre bloques del depósito de talud. La presencia y disposición de las distintas discontinuidades en las rocas de la Formación Mexcala no tiene influencia en los deslizamientos del material.

Las principales afectaciones corresponden a fracturamiento y fallamiento de paredes y pisos. Las familias afectadas son hasta ahora 32 , sin embargo, existe una alta probabilidad de que ésta cantidad aumente.

Los deslizamientos de material son el producto de la interacción entre la pendiente del terreno, el suelo y el depósito de talud. El principal factor detonante es el agua de lluvia, aunque no se descarta que la sismicidad de la región del Río Balsas pueda tener repercusión en la zona.

Se determinaron tres zonas con distinto grado vulnerabilidad, una de riesgo no mitigable, otra de riesgo 
parcialmente mitigable y otra más se considera área segura. La zona de riesgo no mitigable es la que presenta la mayor cantidad de afectaciones, además de la probabilidad de deslizamiento de bloques de la zona denominada La Joya. En esta área es necesario determinar la velocidad de deslizamiento y el análisis detallado de la geometría y continuidad de las grietas. La zona de riesgo parcialmente mitigable incluye al resto de la población que se encuentra asentado sobre el depósito de talud y sobre roca sana que hasta ahora no presentan afectaciones por el deslizamiento de bloques y de suelo. La vulnerabilidad en esta zona debe analizarse con detalle y de manera puntual.

Se recomienda la reubicación de las familias cuyas viviendas se encuentran dentro de la zona de riesgo no mitigable. De manera preliminar se identificaron dos áreas seguras, sin embargo, la limitante del suministro de agua en una y el tamaño en la otra, plantean la necesidad de buscar otras alternativas.

\section{Agradecimientos}

El estudio se realizó a petición las autoridades de Tlacuitlapa y el H. Ayuntamiento del Municipal de Teloloapan, Guerrero 2002-2006. Se agradece la colaboración en las actividades de campo de los compañeros: Angela Aragón Matías, Nayeli Meléndez Colín, Erick Morán Cuevas, Alejandro Roque Ayala, Gilberto Rosas Martínez y Alfonso Sáenz Rivera. En el desarrollo de este trabajo se contó con el apoyo de la población, encabezada por el Prof. Justino Pedro Osorio, el Dr. Celerino Zagal, el Prof. Nicomedez Huerta, el Sr. Cristino Desiderio Sánchez y el Sr. Neri Román. A Luis Arroyo Delgado y Cesar Martínez Serena por su apoyo en la toma y edición de fotografías. A los revisores anónimos que enriquecieron de manera sustancial el presente trabajo. A Barbara Martiny por la revisión del resumen en inglés. De manera muy especial al Dr. Armando García Palomo (q.d.e.p.) por sus comentarios y sugerencias.

\section{Referencias bibliográficas}

Burns, S., Beckstrand, D., Lunney, M., Taylor, J., Robinson, C., Schick, J., 2001, Is your house creeping on a reactivated landslide? (resumen), en GSA Cordilleran Section-97th Annual Meeting: Universal City, C.A.,USA, American Association of Petroleum Geologists, 4085.

Campa, M.F., Coney, P.J., 1983, Tectono-stratigraphic terranes and mineral resource distributions of Mexico: Canadian Journal of Earth Sciences, 20, 1040-1051.

Campa, M.F., 1985, The Mexican thrust belt, en Howell, D.G. (ed.), Tectonostratigraphic terranes of the Circum-Pacific region: CircumPacific Council for Energy and Mineral Resources Earth Sciences Series, 1, 299-313.

Coch, N.K., 1995, Geohazards natural and human: New Jersey. Prentice Hall, Englewood Cliffs.

Dai, F.C., Lee, C.F., Ngai, Y.Y., 2002, Landslide risk assessment and management: an overview: Engineering Geology, 64, 65-87.

Fries, C., 1960, Geología del Estado de Morelos y partes adyacentes de
México y Guerrero, región central meridional de México: Boletín del Instituto de Geología UNAM, 60, 236 p.

Guerrero S.M., Ramírez, E.J., Talavera, M.O., Campa, M.F., 1991, El desarrollo carbonatado del Cretácico Inferior asociado al Arco Teloloapan, noroccidente del Estado de Guerrero (resumen), en Congreso de la Evolución Geológica de México. Sociedad Mexicana de Mineralogía. 67.

Hoek, E., Bray, J., 1996, Rock slope Engineering: London, UK, E \& FN Spon, and imprint of Chapman \& Hall, Third edition..

Instituto Nacional de Estadística y Geografía Informática (INEGI), 1988, Carta topográfica Teloloapan E14A77, escala 1:50 000.

Instituto Nacional de Estadística y Geografía Informática (INEGI), 2005, II Conteo de población y vivienda. www.inegi.gob.mx. Consulta Octubre del 2007.

Morales, S.S., 1989, Estudio paleontológico del Cretácico Inferior basado en gasterópodos, de la parte norte del Estado de Guerrero: Revista de la Sociedad Mexicana de Paleontología, 3 (1), 203-208.

Nelson S.A., 2000, Geology 204. Mass-wasting and mass-wasting processes. Natural Disasters. Tulane University.

Ontiveros, T. G., 1973, Estudio estratigráfico de la porción noroccidental de la Cuenca Guerrero-Morelos: Boletín de la Asociación Mexicana de Geólogos Petroleros 4-6, (25), 190-234.

Pérez-Gutiérrez, R., 1993, Las características del Cinturón Mexicano de Pliegues y Cabalgaduras en el área de Acatempan, Guerrero: Taxco, Guerrero, Escuela Regional de Ciencias de la Tierra, Universidad Autónoma de Guerrero. Tesis Profesional.

Servicio Sismológico Nacional. www.ssn.unam.mx. Consulta NoviembreDiciembre del 2004.

Manuscrito recibido: Septiembre 10, 2007

Manuscrito corregido recibido: Febrero 6, 2008

Manuscrito aceptado: Abril 10, 2008 